\title{
Regional ionosphere modeling using spherical cap harmonics and empirical orthogonal functions over Iran
}

\author{
Mir Reza Ghaffari Razin ${ }^{1} \cdot$ Behzad Voosoghi $^{1}$
}

Received: 27 July 2015/ Accepted: 29 January 2016/Published online: 22 February 2016

(C) Akadémiai Kiadó 2016

\begin{abstract}
The ionosphere is the ionized part of the upper region of the atmosphere extending from 60 to $1500 \mathrm{~km}$ above the earth's surface. In this layer, free electrons are produced during the interaction of extreme ultra violet and $\mathrm{x}$-ray radiation with the upper neutral atmosphere. Knowledge of the ionospheric electron density distribution is important for scientific studies and practical applications. In this paper, a new computerized ionospheric tomography reconstruction technique is developed to estimate electron density profiles over Iran. In this method, a functional based model is used to represent the electron density in space. The functional based model uses empirical orthogonal functions and spherical cap harmonics to describe the vertical and horizontal distribution of the electron density, respectively. The degree and the number of basis functions are chosen so that, the relative error of results is minimized. For this purpose, ionosonde observations $\left(\right.$ Lat. $=35.73^{\circ}$, Lon. $\left.=51.38^{\circ}\right)$ at 2007.04 .03 is used. To apply the method for constructing a 3D-image of the electron density, GPS measurements of the Iranian permanent GPS network (at 2012/08/11) has been used. The modeling region is between 24 to $40 \mathrm{~N}$ and 44 to $64 \mathrm{~W}$. The result of 3D-model has been compared to that of the international reference ionosphere model 2012 (IRI-2012). The analysis conducted in this paper indicates that the choice of spherical cap harmonics to $3\left(\mathrm{~K}_{\max }=3\right)$ and empirical orthogonal function in $3(\mathrm{Q}=3)$, the regional reconstructed error is less than $36 \%$. The results show the advantages of this method in modeling of the ionosphere electron density on local and regional scales.
\end{abstract}

Keywords Ionosphere electron density $\cdot$ Spherical cap harmonics $\cdot$ Empirical orthogonal functions · GPS · IRI2012

Mir Reza Ghaffari Razin

rghaffari@mail.kntu.ac.ir

1 Department of Geodesy and Geomatics Engineering, K. N. Toosi University of Technology,

No. 1346, Vali_Asr Ave., Mirdamad Cr., Tehran, Iran 


\section{Introduction}

The ionosphere is the ionized part of the atmosphere extending from $60 \mathrm{~km}$ to $1500 \mathrm{~km}$ above the earth's surface. It is very important to know ionospheric electron density distribution for scientific studies and practical applications. A global navigation satellite system measurement utilizing global positioning system (GPS) is an effective and valuable tool to study the physical properties of the ionosphere. Due to the large spatial coverage of satellite networks, as well as the availability of permanent measurements, it is possible using observations of these networks to conduct investigations of the ionosphere. Using dual-frequency GPS receivers, slant total electron content (STEC) is computed. STEC contains valuable information about the ionosphere (Seeber 2003). It is a very important quantity used for ionospheric processes.

Since the deactivation of selective availability (SA), the accuracy of the differential positioning with GPS is mostly dominated by the refraction delay of the GPS carrier waves in the ionosphere. That is proportional with STEC. The total electron content represents the number of free electrons between the receiver and the satellite in line of sight. When the STEC is known, it enables computation of ionospheric delay in GPS signals (Zhang et al. 2012). In single-frequency GPS receivers for the removal of ionospheric refraction, TEC values should be determined. TEC can be calculated utilizing ionospheric models. Some of these models provide global coverage while some are regional. Regional models are usually divided into two main categories: one is a grid-based model, such as the satellitebased augmentation system (SBAS), while the other is based on mathematical functions, e.g., the polynomial model (Komjathy 1997), the triangle series model (Georgiadou 1994; Georgiadou and Kleusberg 1988), and the low-degree spherical function model (Wilson et al. 1995). Usually, ionosphere models represent average conditions and often are defined for low altitude ionosphere.

In recent years the idea of using the tomography method to determine the physical properties of the ionosphere is studied. In fact, ionospheric tomography is a reconstruction method using TEC measurements as an input parameter. This technology was first successfully used in medical science and then extended to other applications. The application of the tomographic reconstruction to 3-D modeling of the electron density using radio waves was proposed for the first time by Austen et al. (1988) and applied by Andreeva et al. (1990). So far, many algorithms and methods provided for ionospheric tomography (Hansen et al. 1997; Hernández-Pajares et al. 2000; Colombo et al. 2000; Mitchell and Spencer 2003; Saito et al. 2007; Erturk et al. 2009; Amerian et al. 2010; Wen and Liu 2010, Ghaffari et al. 2015).

Spherical harmonic analysis is a very powerful tool to process complex phenomena over a sphere, such as the ionosphere. To show the electron density profiles with short wavelength (high frequency), high degrees and orders of spherical harmonics will be required. With increasing degree and order of harmonic functions, the number of unknown coefficients will also increase. This leads to an increased demand of computational memory and mathematical calculations. Also the high order of spherical harmonics leads to instability in parameter estimation. In this paper, a method for localization of spherical harmonics has been developed for regional modeling of ionosphere electron density. This model is more accurate for regional modeling and is known as a spherical cap harmonic model (Haines 1988). Spherical cap harmonic analysis has been used in a wide range of applications involving spherical cap geometries, including, for example, mapping electrostatic potential, geomagnetic fields, and ionospheric current 
systems (De Santis et al. 1991, 1992; De Franceschi et al. 1994; Liu et al. 2014). As the basis functions of the spherical harmonics are no longer orthogonal in the local area, the spherical cap harmonic function is introduced. Since the associated Legendre function (where $\mathrm{n}$ and $\mathrm{m}$ are integers) is orthogonal only over the entire sphere, another set of orthogonal functions must be used instead. The new functions that are still Legendre functions of integer order $\mathrm{m}$ but non-integer degree $\mathrm{n}$. The new non-integer Legendre functions and their derivatives have, alternatively, zero value at the edge of a cap with half-angle. Therefore, the new Legendre functions divided into two sets: "even" and "odd". Legendre functions of the odd set are not all orthogonal to those of the even set, but the two sets are separately orthogonal and together provide the basis for a uniformly convergent series for the ionospheric local modeling and its gradient.

The paper is organized as follows: in Sect. 2, the methodology for computation of ionospheric information from GPS observations is presented. Spherical cap harmonic and empirical orthogonal functions are studied in Sect. 3. Parameter estimation and error analysis is explained Sect. 4. Finally, in Sect. 5, the procedure is applied to real GPS data, which were collected from observation sites in Iran.

\section{STEC observations}

The geometry-free linear combination of GPS observations is used to derive the STEC observable. The geometric range, clock-offsets and tropospheric delay are frequency independent and can be eliminated using this combination. The geometry-free linear combinations for pseudorange and carrier phase observations are given as (Kleusberg and Teunissen 1998):

$$
\begin{gathered}
P_{4}=P_{1}-P_{2}=I_{1}-I_{2}+b_{r}^{P}+b_{s}^{P}+\varepsilon_{12}^{P} \\
\Phi_{4}=\Phi_{1}-\Phi_{2}=I_{1}-I_{2}+\lambda_{1} N_{1}-\lambda_{1} N_{2}+b_{r}^{\Phi}+b_{s}^{\Phi}+\varepsilon_{12}^{\Phi}
\end{gathered}
$$

$I_{1}$ and $I_{2}$ are ionospheric delays on $L_{1}$ and $L_{2}$ pseudorange $(m), \lambda_{1}$ and $\lambda_{2}$ are the carrier wavelengths, $N_{1}$ and $N_{2}$ are the carrier phase ambiguities, $b_{r}^{P}$ and $b_{s}^{P}$ are the receiver and the satellite code-delay inter-frequency bias (IFB) (m) respectively, $b_{r}^{\Phi}$ and $b_{s}^{\Phi}$ are the receiver and the satellite phase-delay IFB $(\mathrm{m})$ respectively, $\varepsilon_{12}^{\mathrm{P}}$ is the combination of multipath and measurement noise on $\mathrm{P}_{1}$ and $\mathrm{P}_{2}(\mathrm{~m}), \varepsilon_{12}^{\Phi}$ is the combination of multipath and measurement noise on $\Phi_{1}$ and $\Phi_{2}(\mathrm{~m})$. Using (1) and (2) STEC can be calculated as follows:

$$
\begin{gathered}
\operatorname{STEC}_{P}=\frac{P_{4}}{\beta}=\operatorname{STEC}+B_{r}^{P}+B_{s}^{P}+\varepsilon_{P 4} \\
\operatorname{STEC}_{\Phi}=\frac{\Phi_{4}}{\beta}=-S T E C+N_{\text {arc }}+B_{r}^{\Phi}+B_{s}^{\Phi}+\varepsilon_{\Phi 4}
\end{gathered}
$$

$\mathrm{B}_{\mathrm{r}}^{\mathrm{P}}$ and $\mathrm{B}_{\mathrm{s}}^{\mathrm{P}}$ is the receiver and satellite code-delay IFB in TECU respectively, $\varepsilon_{\mathrm{P} 4}$ is the combination of multipath and measurement noise on $\mathrm{P}_{1}$ and $\mathrm{P}_{2}$ in TECU, $\mathrm{B}_{\mathrm{r}}^{\Phi}$ and $\mathrm{B}_{\mathrm{s}}^{\Phi}$ is the receiver and satellite phase-delay IFB in TECU respectively, $\varepsilon_{\Phi 4}$ is the combination of multipath and measurement noise on $\Phi_{1}$ and $\Phi_{2}$ in TECU. Pseudorange measurements are subject to high noise and multipath effects. As a result, the code-derived STEC $_{P}$ observation is noisy. To reduce the multipath and noise level in the $\mathrm{STEC}_{\mathrm{P}}$ observables, 
the carrier phase measurements are used to compute a more precise relative STEC observable. To obtain ambiguity independent and high precision STEC values, code pseudo-ranges are smoothed using "carrier to code leveling process" (Ciraolo et al. 2007). In this approach, the continuous arcs of $\mathrm{STEC}_{\Phi}$ are adjusted to the mean value of the corresponding code $\mathrm{STEC}_{\mathrm{P}}$ value. The mean value is computed for every continuous arc using:

$$
\left\langle S T E C_{P}+S T E C_{\Phi}\right\rangle=\frac{1}{N} \sum_{i=1}^{N}\left(\operatorname{STEC}_{P}+S T E C_{\Phi}\right)
$$

where $\mathrm{N}$ is the number of continuous measurements contained in the arc. Subtracting Eq. (4) from (5), the smoothed STEC can be derived:

$$
S T E C_{\text {smoothed }}=\left\langle S T E C_{P}+S T E C_{\Phi}\right\rangle-S T E C_{\Phi}=S T E C+\left(B_{r}^{P}+B_{s}^{P}\right)+\varepsilon_{P 4}
$$

\section{Model development}

For the local reconstruction of ionosphere electron density, we can use spherical cap harmonics and empirical orthogonal functions as base functions.

\subsection{Spherical cap harmonics (SCHs)}

For applications where data are limited to a certain area such as a cap or a small part of the sphere, Legendre polynomials do not provide a good fit. These basis functions are of a global nature and for a specific area they are not suitable. Therefore, spherical harmonics are not suitable for high-resolution modeling and are only used in long-wavelength modeling applications. Legendre polynomials are orthogonal on a sphere but not for a specific area. The bias introduced through the data cut-off at the boundaries (Gibbs phenomenon) is another weakness in the regional modeling of the ionosphere using spherical harmonic functions (Schmidt 2007).

Haines (1988) introduced a technique called spherical cap harmonic analysis (SCHA) to solve this particular problem. The technique can be used to model a general function on a cap-like region by new functions that are still Legendre functions of integer order $\mathrm{m}$ but non-integer degree $\mathrm{n}$. A spherical cap is a part of the sphere with a co-latitude range from zero to $\theta_{0}$, as shown in Fig. 1 .

Fig. 1 A spherical cap with a radius (a) and a half angle $\left(\theta_{0}\right)$

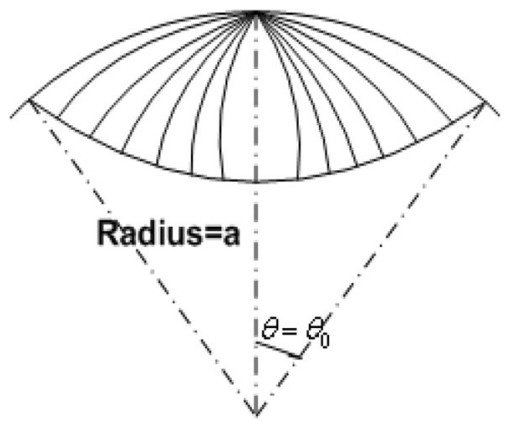


The spherical cap harmonic model for mapping the regional TEC can be expressed as:

$$
f(\lambda, \theta)=\sum_{k=0}^{K_{\max }} \sum_{m=0}^{k}\left[a_{k}^{m} \cos (m \lambda)+b_{k}^{m} \sin (m \lambda)\right] P_{n_{k}}^{m}(\cos \theta)
$$

which corresponds to a spherical expansion developed over a cap-like region in a new reference system with the north pole at the centre of the spherical cap. $\mathrm{K}_{\max }$ is the maximum degree at which the expansion is truncated. The first step in $\mathrm{SCH}$ analysis is to convert the geographic colatitudes $(\theta)$ and longitudes $(\lambda)$ of the data from old values in the normal geographic system to new values relative to the new pole (spherical cap coordinate system). The spherical cap coordinate system is an earth-centered coordinate system. The pole of the spherical cap is chosen to define the coordinate system. Figure 2 illustrates the relationship between the geographic coordinate system and spherical cap coordinate system (Liu et al. 2009).

If the geographic coordinates of the new pole in the old system are $\theta_{0}$ and $\lambda_{0}$ then the new coordinates $\left(\theta_{\mathrm{C}}, \lambda_{\mathrm{C}}\right)$ of any point $\mathrm{Q}(\theta, \lambda)$ can be computed using the following:

$$
\begin{gathered}
\cos \left(\theta_{c}\right)=\cos \left(\theta_{0}\right) \cos (\theta)+\sin \left(\theta_{0}\right) \sin (\theta) \cos \left(\lambda-\lambda_{0}\right) \\
\tan \left(\pi-\lambda_{c}\right)=\frac{\sin (\theta) \sin \left(\lambda-\lambda_{0}\right)}{\sin \left(\lambda_{0}\right) \cos (\theta)-\cos \left(\theta_{0}\right) \sin (\theta) \cos \left(\lambda-\lambda_{0}\right)}
\end{gathered}
$$

\subsection{Empirical orthogonal functions (EOFs)}

The vertical resolution in ionospheric tomography is generally not very good. Therefore, a priori information is necessary to form the vertical basis functions that span the entire space in the vertical direction. This is accomplished using empirical orthogonal functions (EOFs). EOFs analysis is a powerful tool for data analysis and reduction of data dimensions. EOFs are derived from empirical data of the ionospheric electron density, such as the international reference ionosphere (IRI) model. Using such functions, the vertical profiles of electron density are obtained. Having the samples of the density profile obtained at different times and heights, the matrix of electron density profile could be formed as:

Fig. 2 Coordinate transformation between geographic coordinate system and spherical cap coordinate system

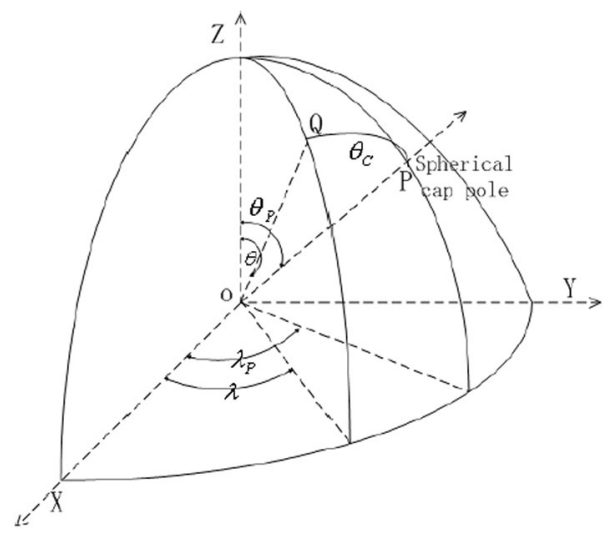




$$
G_{M \times N}=\left[\begin{array}{ccc}
N_{e}\left(\lambda_{1}, \phi_{1}, h_{1}, t_{1}\right) & \cdots & N_{e}\left(\lambda_{1}, \phi_{1}, h_{1}, t_{N}\right) \\
\vdots & \ddots & \vdots \\
N_{e}\left(\lambda_{i}, \phi_{j}, h_{k}, t_{1}\right) & \cdots & N_{e}\left(\lambda_{i}, \phi_{j}, h_{k}, t_{N}\right)
\end{array}\right]
$$

where $\mathrm{M}=\mathrm{i} \times \mathrm{j} \times \mathrm{k}$ is the number of voxels, and $\mathrm{N}$ is the number of times series. To obtain the required EOFs, singular value decomposition (SVD) can be used:

$$
G_{M \times N}=U_{M \times M} S_{M \times N} V_{N \times N}^{T}
$$

where $\mathbf{U}$ and $\mathbf{V}$ are orthogonal matrices with columns that span the data and model spaces respectively, and $\mathbf{S}$ is a diagonal matrix. If the singular values of the matrix $\mathbf{S}$ will be sorted in descending order, the eigen-vector corresponding to the largest singular value is a first EOF. Figure 3 shows the first 3 EOFs extracted from electron density profiles obtained from IRI-2012 for April 3rd, 2007.

According to these results, the first three eigenvalues of matrix $\mathbf{G}$ contain 98 percent of the total variations of the electron density. Therefore, the first three EOFs have been used for modeling the vertical variations of the electron density in this research.

\subsection{Development of 3-D model}

The total electron content represents the total number of electrons in a column along the direction of a satellite (sv) to a receiver (rx) (Coster et al. 2003). It can be expressed as:

$$
S T E C=\int_{r x}^{s v} N_{e}(\lambda, \phi, h) d s
$$

Combining spherical cap harmonic functions and the EOFs, the electron density distribution $\mathrm{N}_{\mathrm{e}}(\lambda, \varphi, \mathrm{h})$ can be expressed as follows:

$$
N_{e}(\lambda, \phi, h)=\sum_{q=1}^{Q} \sum_{k=0}^{K_{\max }} \sum_{m=0}^{k}\left[a_{k q}^{m} \cos (m \lambda)+b_{k q}^{m} \sin (m \lambda)\right] P_{n_{k}}^{m}(\cos \theta) Z_{q}(h)
$$

where $\mathrm{Z}_{\mathrm{q}}(\mathrm{h})$ represents the empirical orthogonal function, $\mathrm{Q}$ is the order of EOFs, $a_{k q}^{m}$ and $b_{k q}^{m}$ are the tomography model coefficients that characterize the field of the ionosphere and

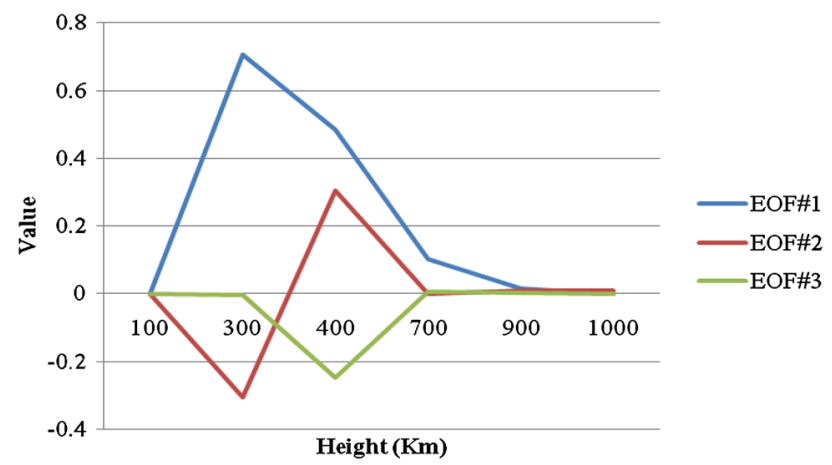

Fig. 3 First three EOFs derived from IRI-2012 model 
the unknowns to be estimated in the model. Combining Eq. (12) and (13) results in the fundamental observation equation in the function based 3-D reconstruction of the electron density as:

$$
S T E C_{\text {smoothed }}=\int_{r x}^{s v} \sum_{q=1}^{Q} \sum_{k=0}^{K_{\max }} \sum_{m=0}^{k}\left[a_{k q}^{m} \cos (m \lambda)+b_{k q}^{m} \sin (m \lambda)\right] P_{n_{k}}^{m}(\cos \theta) Z_{q}(h) d s
$$

Equation (14) is expressed in a matrix form as:

$$
\overrightarrow{\mathbf{d}}=\mathbf{G} \cdot \overrightarrow{\mathbf{m}}+\overrightarrow{\mathbf{v}}
$$

where $\mathbf{d}$ is the observation vector, $\mathbf{G}$ contains the basis functions generated using EOFs and $\mathrm{SCH}$ expansion, $\mathbf{m}$ contains the tomography model coefficients and $\mathbf{v}$ is the observation noise vector.

\section{Parameter estimation}

The design matrix $\mathbf{G}$ in Eq. (15) is nearly singular (large condition number with respect to singular values). It is difficult to invert it without round-off errors affecting the solution and even causing it to converge to the wrong solution. Therefore, ionospheric tomography is part of the family of inverse problems. The ionospheric tomography inverse problem is often ill-posed, which is characterized by instability, non-uniqueness and even non-existence of the solution. Therefore, ordinary inversion techniques are not efficient and special inversion techniques must be considered in ionospheric tomography. Generalized Tikhonov regularization is one of the most common methods for regularization that satisfies the following (Mead 2007):

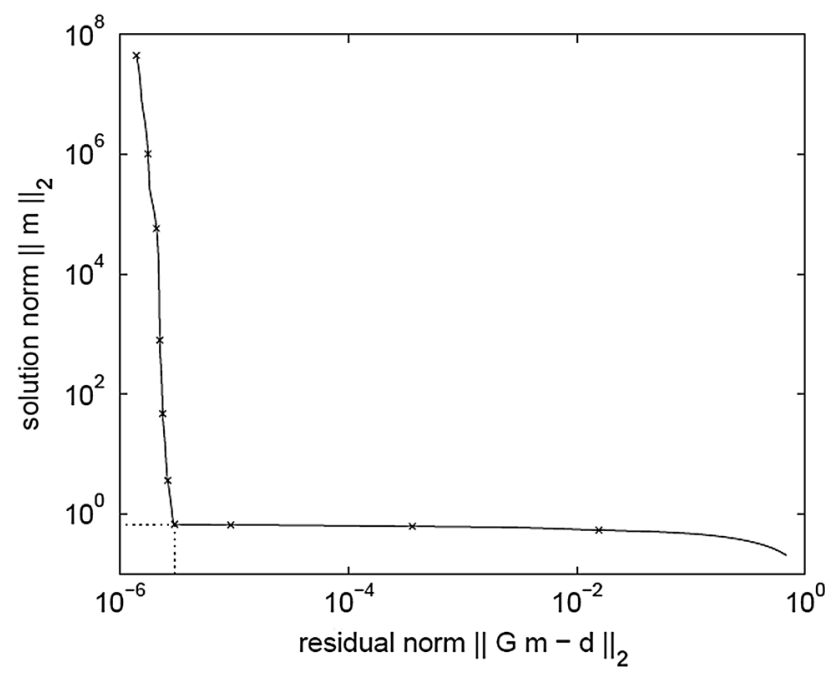

Fig. 4 The L-curve 


$$
\min \left(\|\overrightarrow{\mathbf{V}}\|_{P_{d}}^{2}+\left\|\overrightarrow{\mathbf{V}}_{\mathbf{X}}\right\|_{P_{m}}^{2}\right)=\min \left(\|\overrightarrow{\mathbf{d}}-\mathbf{G} \overrightarrow{\mathbf{m}}\|_{P_{d}}^{2}+\alpha^{2}\left\|\left(\hat{\mathbf{m}}-\overrightarrow{\mathbf{m}}_{\mathbf{0}}\right)\right\|_{P_{m}}^{2}\right)
$$

where $\mathrm{P}_{\mathrm{d}}$ and $\mathrm{P}_{\mathrm{m}}$ are the inverse of the variance-covariance matrix of the observations, the initial parameter estimates $\overrightarrow{\mathbf{m}}_{\mathbf{0}}$ and $\alpha$ regularization parameter, respectively. Using (16), the unknown parameters can be obtained as follows:

$$
\hat{\mathbf{m}}=\overrightarrow{\mathbf{m}}_{0}+\left(\mathbf{G}^{T} \mathbf{P}_{d} \mathbf{G}+\alpha^{2} \mathbf{P}_{m}\right)^{-1} \mathbf{G}^{T} \mathbf{P}_{d}\left(\overrightarrow{\mathbf{d}}-\mathbf{G} \overrightarrow{\mathbf{m}}_{0}\right)
$$

\subsection{Selection of regularization parameter}

In regularized solutions the selection of the regularization parameter is very important. If this parameter is very small, estimated unknowns are affected by noise, and also if the regularization parameter is chosen too large, the solution will be too smooth. The L-curve method is one of the most common tools for selecting a regularization parameter. The regularization parameter which corresponds to the corner point of the curve is an optimum estimate for this parameter. Figure 4 shows the generic form of the L-curve.
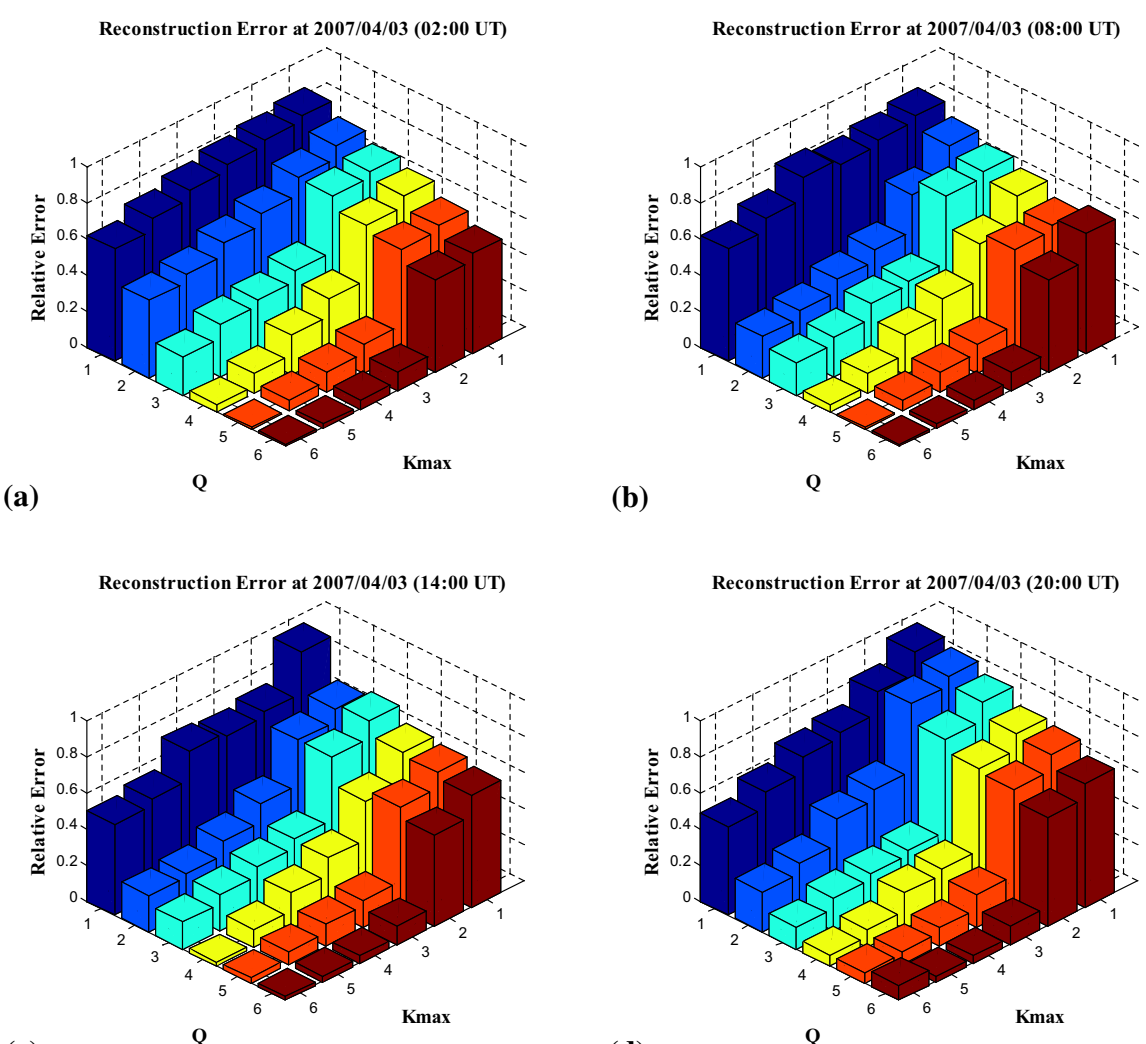

(c)

(b)

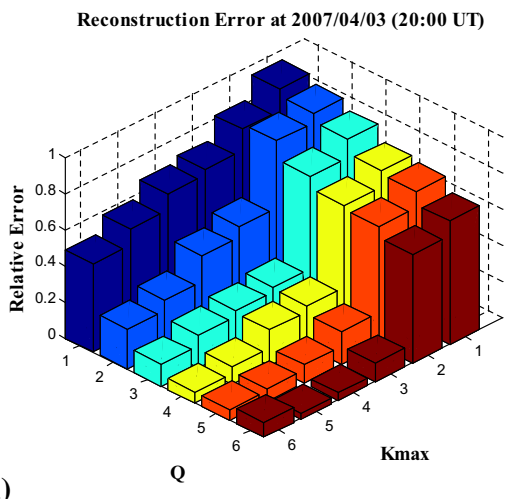

Fig. 5 Reconstruction error (Re) for 03-march of years 2007 at different UT time intervals. a Reconstruction error (Re) for 03-March 2007 at UT 02:00. b Reconstruction error (Re) for 03-March 2007 at UT 08:00. c Reconstruction error (Re) for 03-March 2007 at UT 14:00. d Reconstruction error (Re) for 03-March 2007 at UT 20:00 
Table 1 Error statistics using different $\mathbf{K}_{\mathbf{m a x}}$ and $\mathbf{Q}$

\begin{tabular}{|c|c|c|c|c|c|c|c|c|c|}
\hline \multirow{2}{*}{$\begin{array}{l}\mathbf{K}_{\max } \\
\text { and } \\
\mathbf{Q}\end{array}$} & \multicolumn{4}{|c|}{ Relative error $(\%)$} & \multicolumn{4}{|c|}{ Condition number } & \multirow{2}{*}{$\begin{array}{l}\text { Number of } \\
\text { unknown }\end{array}$} \\
\hline & $\begin{array}{l}\mathrm{UT}= \\
02: 00\end{array}$ & $\begin{array}{l}\mathrm{UT}= \\
08: 00\end{array}$ & $\begin{array}{l}\mathrm{UT}= \\
14: 00\end{array}$ & $\begin{array}{l}\mathrm{UT}= \\
20: 00\end{array}$ & $\begin{array}{l}\mathrm{UT}= \\
02: 00\end{array}$ & $\begin{array}{l}\mathrm{UT}= \\
08: 00\end{array}$ & $\begin{array}{l}\mathrm{UT}= \\
14: 00\end{array}$ & $\begin{array}{l}\mathrm{UT}= \\
20: 00\end{array}$ & \\
\hline$(2,2)$ & 78 & 69 & 75 & 81 & 876 & 912 & 821 & 978 & 18 \\
\hline$(3,3)$ & 36 & 41 & 39 & 28 & 973 & 1054 & 984 & 901 & 48 \\
\hline$(4,4)$ & 29 & 32 & 24 & 25 & 4698 & 5137 & 4238 & 4312 & 100 \\
\hline$(5,5)$ & 18 & 12 & 11 & 10 & 8231 & 8129 & 8024 & 7897 & 216 \\
\hline$(6,6)$ & 7 & 9 & 6 & 9 & 12,349 & 12,798 & 12,276 & 12,694 & 343 \\
\hline
\end{tabular}

\subsection{Error evaluation}

In this paper, to evaluate the results of the proposed method, reconstructed electron densities are compared with electron densities obtained from an ionosonde station $\left(\right.$ Lat. $=35.73^{\circ}$, Lon. $\left.=51.38^{\circ}\right)$. This station is located at the Tehran Institute of Geophysics. A relative and absolute error is used to evaluate results accuracy as follows:

$$
\operatorname{Re}=\frac{\left\|N_{e}^{\text {Estimated }}-N_{e}^{\text {Truth }}\right\|}{\left\|N_{e}^{\text {Truth }}\right\|}
$$

where $\mathrm{N}_{\mathrm{e}}^{\text {Estimated }}$ is the estimated electron density using proposed method and $\mathrm{N}_{\mathrm{e}}^{\text {Truth }}$ is electron density obtained from ionosonde measurements.

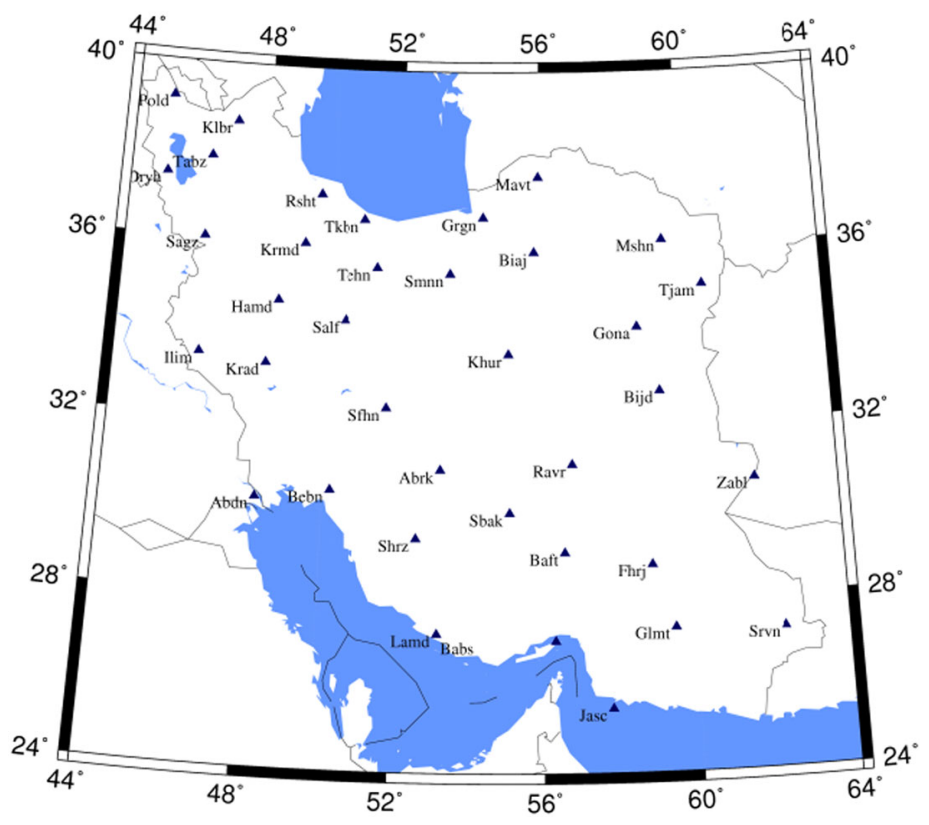

Fig. 6 Spatial distribution of the GPS stations used in this study 

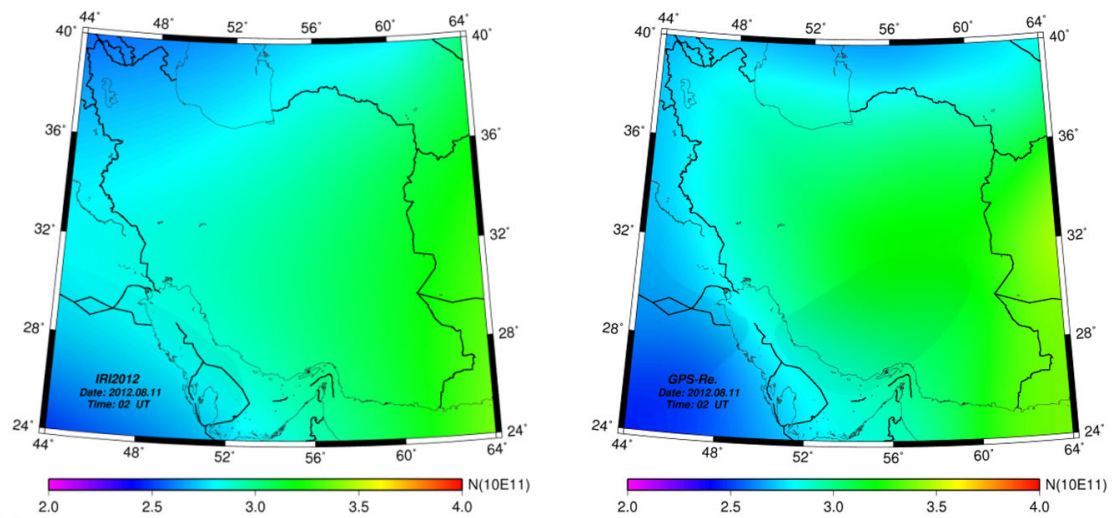

(a)
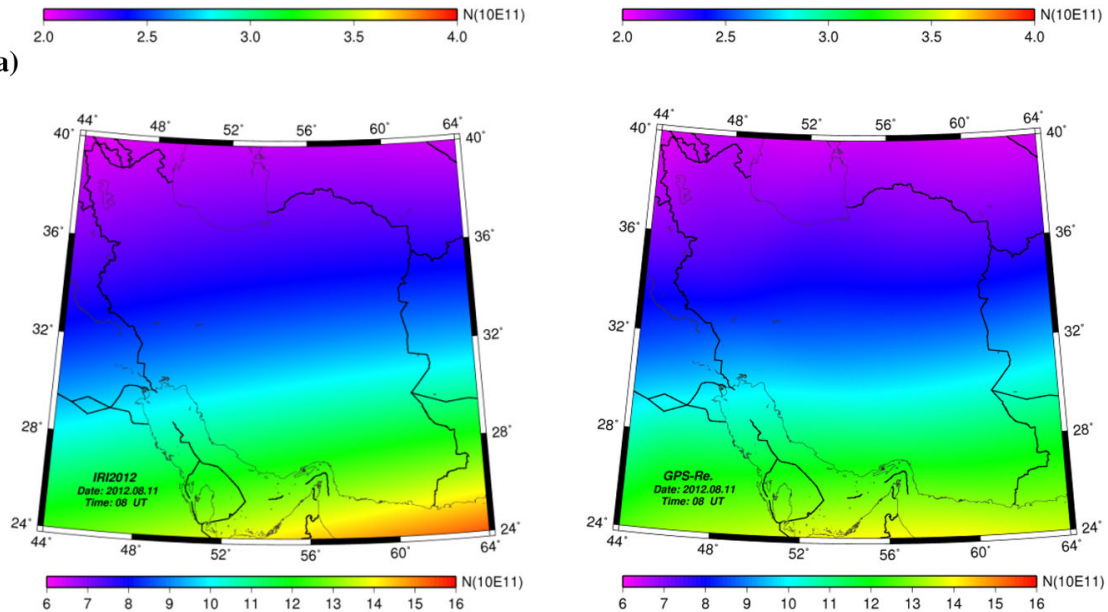

(b)
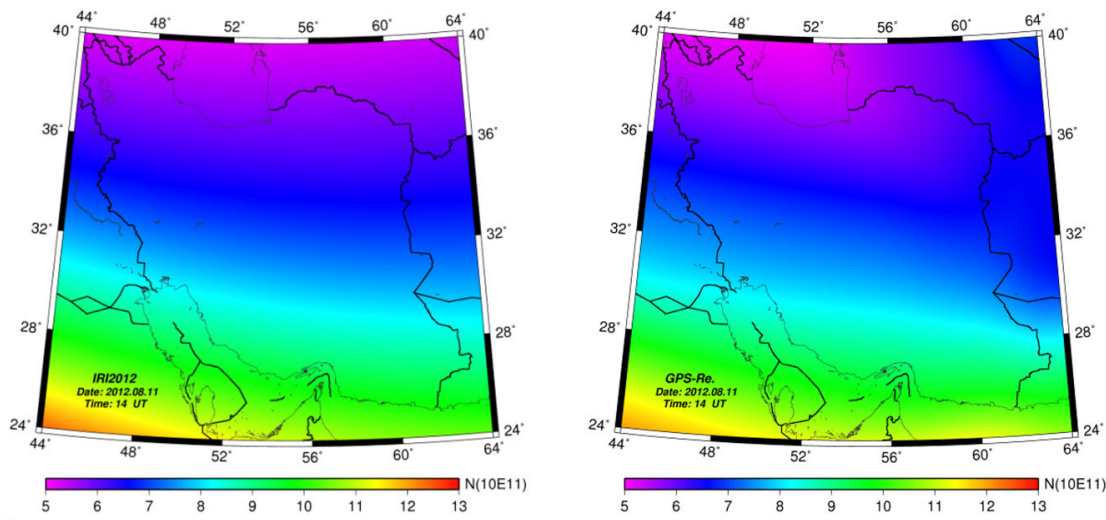

(c)

Fig. 7 Comparison of horizontal variations of the electron density for four different time intervals during 2012.08.11, IRI2012 model (left panel) and reconstructed model (right panel). a comparison of horizontal variations of the electron density at 02 UT in 2012.08.11, IRI2012 model (left) and reconstructed model (right). b comparison of horizontal variations of the electron density at 08 UT in 2012.08.11, IRI2012 model (left) and reconstructed model (right). c comparison of horizontal variations of the electron density at 14 UT in 2012.08.11, IRI2012 model (left) and reconstructed model (right). d comparison of horizontal variations of the electron density at 20 UT in 2012.08.11, IRI2012 model (left) and reconstructed model (right) 

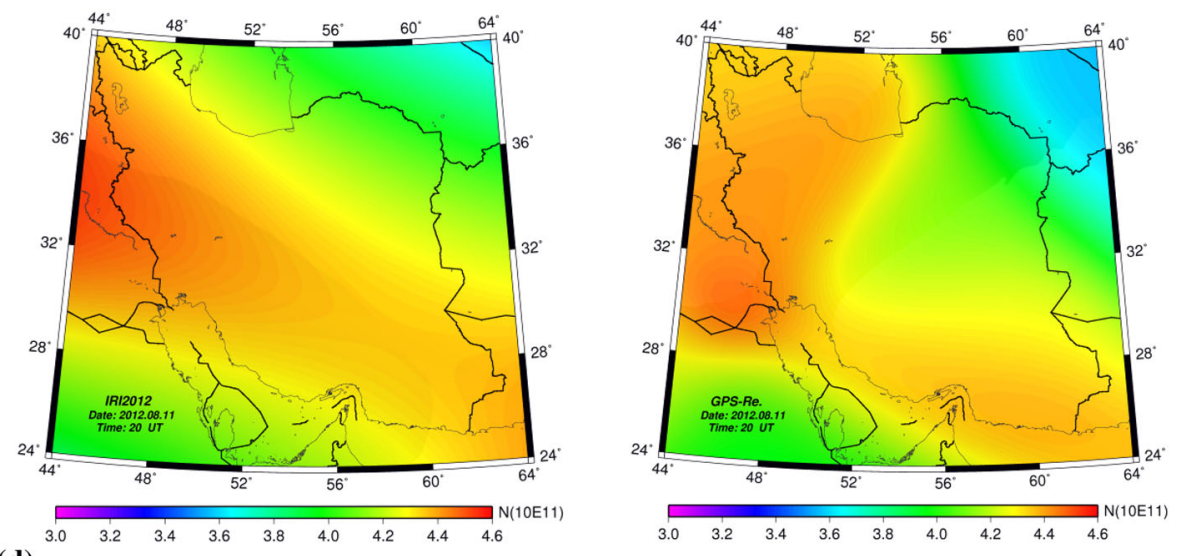

(d)

Fig. 7 continued

\subsection{Parameter optimization}

The number of unknown parameters in ionosphere modeling using tomography method is determined by the degree of the spherical cap harmonics $\left(\mathbf{K}_{\mathbf{m a x}}\right)$ and the number of empirical orthogonal functions (Q). The values of $\mathbf{K}_{\max }$ and $\mathrm{Q}$ are proportional to the number of unknown parameters. Figure 5 shows plots of error analysis at different UT time intervals.

The results confirm the fact that the electron density profiles can be described more accurately if higher $\mathbf{K}_{\mathbf{m a x}}$ and $\mathbf{Q}$ are used. The first EOF, which is the most dominant function, represents a mean electron density profile. The higher order EOF's allow the variation of the profile from the mean. However, their significance decreases gradually to a point where including additional EOF does not add any significant information. The higher order EOF's will increase the condition number of the design matrix and make the inversion technique unstable. This is demonstrated in Table 1.

Different $\mathbf{K}_{\max }$ and $\mathbf{Q}$ values are used and the statistics of the errors corresponding to the solution using these values are listed. As the number of EOF increases the condition number of the design matrix increases and as a result the errors become larger. Therefore, the minimum optimum values for both $\mathbf{K}_{\mathbf{m a x}}$ and $\mathbf{Q}$ over the Iran region for this investigation are chosen as 3 .

\section{Numerical results}

Iran geodynamic studies started in 1998 to monitor the variations in the Earth's crust and tectonic movements. A permanent GPS network was designed and implemented gradually since 2004 to investigate the mechanisms of active faults in Iran. This network currently has 120 permanent GPS stations in the initial phase. Average distance between dense parts is about 25 to $30 \mathrm{~km}$. From these 120 stations, 37 stations are selected for modeling ionospheric tomography over Iran since August 11, 2012. Figure 6 shows the spatial distribution of these stations. In this figure, black triangles indicate stations used in this study. 


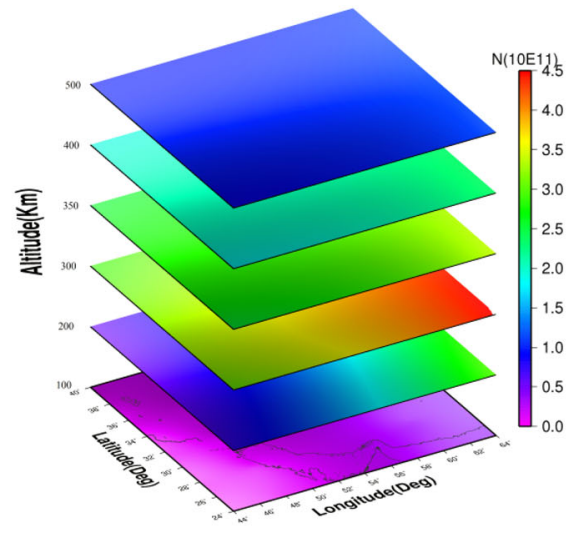

(a)

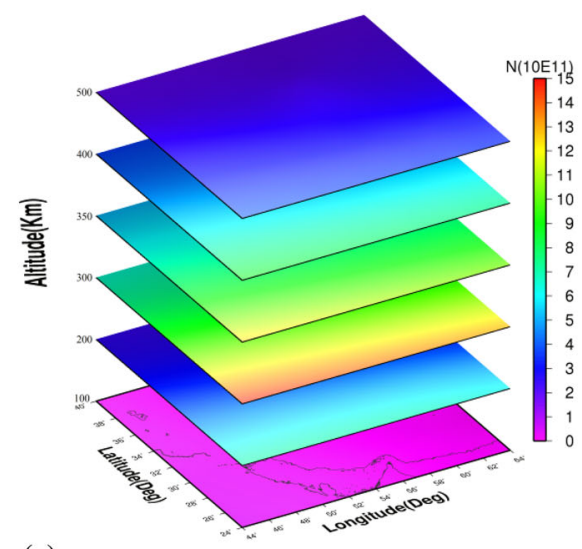

(c)

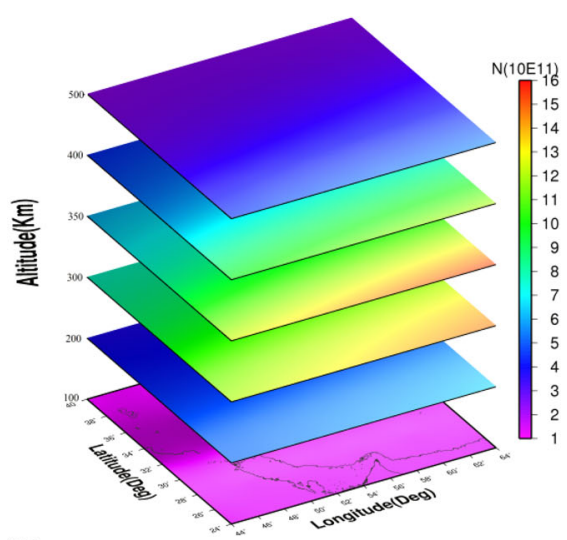

(b)

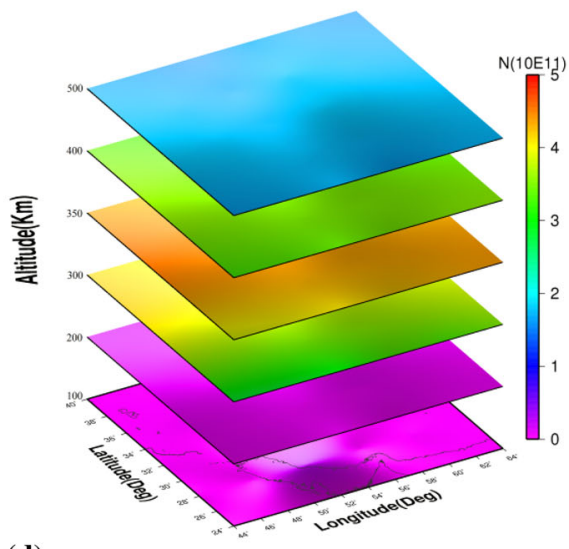

(d)

Fig. 8 The model estimates of electron density at six height layer in four times $(02,08,14$ and 20 UT) at 2012.08.11. a The model estimates of electron density at six height layer in 2012.08.11 and 02 UT. b The model estimates of electron density at six height layer in 2012.08.11 and 08 UT. c The model estimates of electron density at six height layer in 2012.08.11 and 14 UT. d The model estimates of electron density at six height layer in 2012.08.11 and 20 UT

To analyze the behavior of the ionosphere electron density, GPS observations in onehour intervals is used. In this period, ionosphere electron density will be varying in both horizontal and vertical directions. A very important point is that, the purpose of this paper is to evaluate the ability of proposed method in modeling of ionosphere spatial variations. Therefore, ionosphere time variation will not be considered. As a result of all analyzes performed in this section is to describe the spatial variability of the ionosphere. In this paper, spherical cap model is centered at latitude $32^{\circ}$ and longitude $54^{\circ}$ with a half-cap angle of $15^{\circ}$. The model estimates of the electron density for four different times $(02,08$, 14 and $20 \mathrm{UT}$ ) at altitude $\mathrm{h}=350 \mathrm{~km}$ are shown in Fig. 7. All results obtained from our reconstruction method were compared with the IRI2012 model values. 
Fig. 9 IRI (circles) and estimated (dash-line) electron density $\left(10^{11}\right.$ electrons $\left./ \mathrm{m}^{3}\right)$ as a function of altitude

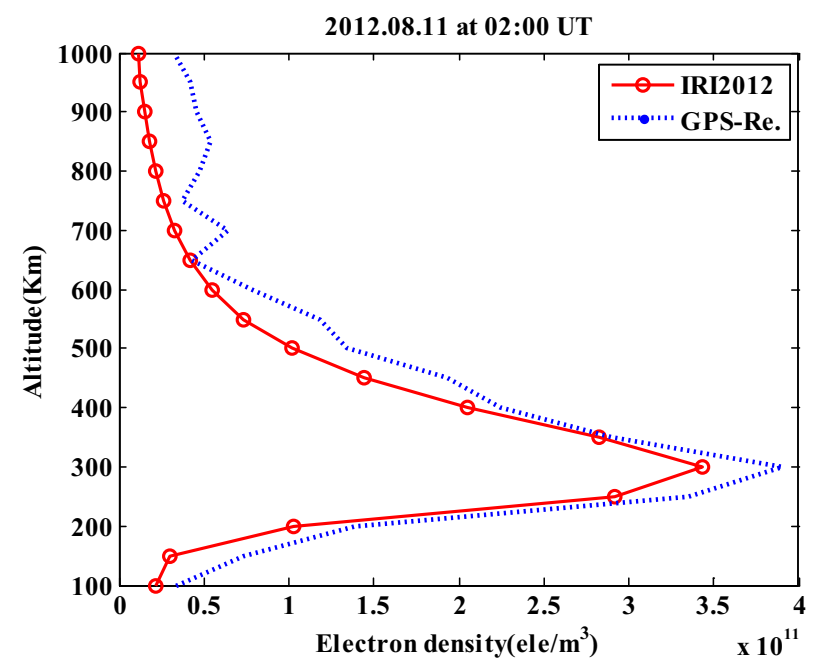

It is essential that the IRI model uses the ionosonde measurements to determine the electron density profiles. In a region like Iran, there is only one ionosonde station. Therefore, in these areas, the IRI model uses interpolation methods to show electron density variations resulting in model values to exhibit large uncertainties. Hence the need to development a model based on GPS measurements from local networks in areas like Iran is absolutely essential.

When using the current model, it is possible to estimate the ionosphere electron density at different heights. Figure 8 shows results from this model at six altitude layers in 2012.08.11. Figures have been drawn at four times (02, 08, 14 and $20 \mathrm{UT})$. According to the results in Fig. 8, ionosphere electron density variations are the highest value in the range of 300-400 km. Unlike 2-D ionosphere models with a fixed height for ionospheric variations, a 3-D model is able to determine electron density variations at different altitudes.

The height profile of the electron density at the position of the Iranian ionosonde $\left(\right.$ Lat. $=35.73^{\circ}$, Lon. $\left.=51.38^{\circ}\right)$ is given in Fig. 9. In this figure, circles are the IRI 2012 extracted data in $10 \mathrm{~km}$ intervals. Illustrated dash-line at this figure gives the tomographic estimate of electron density.

\section{Conclusions}

Due to the characteristics of spherical harmonics including their definition on the sphere and also the orthogonally of Legendre polynomials, they are not suitable for regional modeling applications. For this purpose, a new 3-D computerized ionosphere tomography (CIT) technique using GPS measurements has been developed. The technique uses a combination of spherical cap harmonics (SCH) and empirical orthogonal functions (EOF) to describe the electron density distribution. The spherical cap harmonics describe the electron distribution horizontally while the empirical orthogonal functions describe and constrain the electron density distribution vertically. The analysis for GPS network presented in this paper, shows that using $\mathbf{K}_{\mathbf{m a x}}=3, \mathrm{Q}=3$ (48 parameters) ionospheric parameters can model the ionosphere layers. The data analysis shows that the latitudinal 
sections of the electron density in ionosphere obtained from the 3-D technique support the expected time and height variations in the electron density. Moreover, these findings show that the height of maximum electron density changes during the day and night. This confirms the efficiency of the developed multi-layer model in comparison to the traditional single-layer ones.

\section{References}

Amerian Y, Mashhadi Hossainali M, Voosoghi B, Ghaffari MR (2010) Tomographic reconstruction of the ionospheric electron density in term of wavelets. J Aerosp Sci Technol 7(1):19-29

Andreeva ES, Galinov AV, Kunitsyn VE, Mel'nichenko YA, Tereshchenko ED, Filimonov MA, Chernykov SM (1990) Radio tomographic reconstructions of ionization dip in the plasma near the Earth. J Exp Theor Phys Lett 52:145-148

Austen JR, Franke SJ, Liu CH (1988) Ionospheric imaging using computerized tomography. Radio Sci 23(3):299-307

Ciraolo L, Azpilicueta F, Brunini C, Meza A, Radicella SM (2007) Calibration errors on experimental slant total electron content (TEC) determined with GPS. J Geod 81(2):111-120. doi:10.1007/s00190-0060093-1

Colombo OL, Hernandez-Pajares M, Juan JM, Snaz J (2000) Ionospheric tomography helps resolve GPS ambiguities on the fly at distances of hundreds of kilometers during increased geomagnetic activity. Presented at the Position location and navigation 159 symposium, IEEE 2000

Coster AJ, Foster J, Erickson P (2003) Monitoring the ionosphere with GPS. Space Weather GPS World 14(5):42-49

De Franceschi G, De Santis A, Pau S (1994) Ionospheric mapping by regional spherical harmonic analysis: new developments. Adv Space Res 14(12):61-64

De Santis A, De Franceschi G, Zolesi B, Pau S, Cander R (1991) Regional mapping of the critical frequency of the F2 layer by spherical cap harmonic expansion. Ann Geophys 9:401-406

De Santis A, De Franceschi G, Zolesi B, Pau S, Cander R (1992) Regional modelling and mapping of the ionospheric parameters by spherical cap harmonic expansion. Adv Space Res 12(6):279-282

Erturk O, Arikan O, Arikan F (2009) Tomographic reconstruction of the ionospheric electron density as a function of space and time. Adv Space Res 43(11):1702-1710

Georgiadou Y (1994) Modeling the ionosphere for an active control network of GPS station, vol 7., LGRseriesDelft Geodetic Computing Centre, Delft

Georgiadou Y, Kleusberg A (1988) on the effect of ionospheric delay on geodetic relative GPS positioning. Manuscripta Geodaetia 13:1-8

Ghaffari MR, Voosoghi B, Mohammadzadeh A (2015) Efficiency of artificial neural networks in map of total electron content over Iran. Acta Geod Geophys. doi:10.1007/s40328-015-0143-3

Haines GV (1988) Computer programs for spherical cap harmonic analysis of potential and general fields. Comput Geosci 14(4):413-447

Hansen AJ, Walter T, Enge P (1997), Ionospheric correction using tomography. In: Proceeding of 10th international technical meeting of the satellite division of the institute of navigation, ION GPS-97, Portland, 16-19 Sep

Hernández-Pajares M, Juan JM, Sanz J, Colombo OL (2000) Application of ionospheric tomography to realtime GPS carrier-phase ambiguities resolution, at scales of 400-1000 km and with high geomagnetic activity. Geophys Res Lett 27:2012

Kleusberg A, Teunissen PJG (1998) GPS for geodesy. Springer, Berlin

Komjathy A (1997) Global ionospheric total electron content mapping using the global positioning system. Ph.D. dissertation, Department of Geodesy and Geomatics Engineering, Technical Report No. 188, University of New Brunswick, p 248

Liu J, Chen R, Kuusniemi H, Wang Z, Zhang H, Yang J (2009) Mapping the regional ionospheric TEC using a spherical cap harmonic model and IGS products in high latitudes and the arctic region. Proceedings of IAIN 2009 World Congress, Stockholm

Liu J, Chen R, An J, Wang Z, Hyyppa J (2014) Spherical cap harmonic analysis of the Arctic ionospheric TEC for one solar cycle. J Geophys Res Space Phys 119:601-619. doi:10.1002/2013JA019501

Mead JL (2007) Parameter estimation: a new approach to weighting a priori information. J Inv Ill-Posed Prob 15:1-21 
Mitchell CN, Spencer PSJ (2003) A three-dimensional time dependent algorithm for ionospheric imaging using GPS. Ann Geophys 46:687-696

Saito A, Teraishi S, Ueno G, Fujita N, Tsugawa T (2007) GPS ionospheric tomography over Japan with constrained least-squares method. Eos Trans. AGU, 88(52), Fall Meet Suppl, Abstract SA13A-1061

Schmidt M (2007) Wavelet modelling in support of IRI. Adv Space Res 39(5):932-940

Seeber G (2003) Satellite geodesy: foundations, methods and applications, 2nd edn. Walter de Gruyter, Berlin, p 531

Wen D, Liu S (2010) A new ionospheric tomographic algorithm constrained multiplicative algebraic reconstruction technique (CMART). J Earth Syst Sci 119(4):489-496

Wilson BD, Mannucci AJ, Edwards CD (1995) Subdaily northern hemisphere ionospheric maps using an extensive network of GPS receivers. Radio Sci 30:639-648

Zhang AED, Ridley AJ, Xiao Z, Hao Y (2012) A global model: empirical orthogonal function analysis of total electron content 1999-2009 data. J Geophys Res 117:A03328. doi:10.1029/2011JA017238 